UFIFT-HEP-92-26

\title{
Point-Like Interactions in String Theory Induced by 2-D Topological Gravity
}

\author{
Zongan $\mathrm{Qiu}^{\ddagger}$ \\ Department of Physics \\ University of Florida \\ Gainesville, FL 32611
}

\begin{abstract}
We consider a string theory with two types of strings with geometric interaction. We show that the theory contains strings with constant Dirichlet boundary condition and those strings are glued together by 2-d topological gravity with macroscopic boundaries. A light-cone string field theory is given and the theory has interactions to all orders.
\end{abstract}

$10 / 92$

$\ddagger$ email: qiu@ufhepa.phys.ufl.edu, qiu@ufhepa.bitnet 


\section{Introduction}

It is well known that the bosonic string theory [1] is not a consistent theory due to the existence of tachyon. Physicists have long suspected the tachyon signals that one considers the theory in the wrong vacuum. One of the goals of formulating string field theory is to discover the true vacuum of string theory. Work in this direction so far yields few insight due to our lack of understanding of closed string field theory. Moreover string field theory to date is formulated to generate the first quantized amplitudes, and is therefore limited by the problems of the first quantized theory. The question of whether there is a consistent $d>1$ bosonic string theory is a fundamental one and deserves further exploration. A similar question also appears in high temperature superstring, where the Hagedorn temperature can be thought of as the appearance of tachyon in the compactified time[2]. Atick and Witten have further argued that the tachyon signals a phase transition in string theory. The tachyon problem has been a mystery in string theory.

Recently, much progress has been made in understanding another class of string theories, non-critical string theories of $d \leq 1[3]$. These theories are consistent bosonic strings without a tachyon. Furthermore, they can be understood in term of hermitian matrix models which give a non-perturbative definition of the theories [4]. Some of the $d \leq 1$ strings are purely topological theories, e.g. two-dimensional Topological Gravity (TG) with $c=-2$ [5] [6]. Many researchers in the field suspect $d \leq 1$ strings are related in some way to critical string theory with $d=26$. But the exact relationship of $d \leq 1$ non-critical strings with critical string theory has not been demonstrated.

In this paper we discuss a string theory that incorporate non-critical $d \leq 1$ strings into critical bosonic string theory. In the first quantized language, the amplitude of the string theory has new contributions from "colored" Riemann surfaces, black and white in our case, which come from interactions between two types of strings. The white region represents ordinary bosonic string with suitable boundary condition and the black region the non-critical strings. In fact, we will only consider a special case where there is a different black string for each space-time point and each one is given by TG. Therefore in the calculation of amplitudes in the theory one not only has to sum over all surfaces but also has to sum over the coloration and all possible black strings as well.

We will describe the gauge fixed theory in this paper and postpone the gauge invariant formulation to later publication. Nevertheless, some formal properties of the gauge invariant theory will be addressed. The gauge fixed theory is a string theory containing 
strings with constant Dirichlet Boundary Condition(DBC). Some general properties of the theory with DBC was studied by Green and Shapiro[7]. But in our theory the point like object comes from TG. The interactions are given by string geometrical interactions and determined to all orders.

The organization of the paper is as follows. In section 2 . we will describe the first quantized theory and review the relevant results of the TG. In section 3, the main ingredients needed to describe our theory are discussed. A string field theory in light-cone gauge is given in section 4 . Various issues related to our results are addressed in section 5 .

\section{The Configuration Space of String Theory and 2-D Topological Gravity}

The Polyakov description of string theory starts with the function integral

$$
Z=\int \mathcal{D} g \mathcal{D} X e^{-S[g, X]}
$$

with the Euclidean action

$$
S[g, X]=\frac{1}{8 \pi} \int d^{2} \xi \sqrt{g}\left(g^{a b} \partial_{a} X^{\mu} \partial_{b} X_{\mu}-4 \mu_{0}\right)
$$

where $X_{\mu}(\xi)$ is the location of the surface in space-time.

Before we proceed further, we would like to argue that the function integral (2.1) contains contribution where the whole world sheet is mapped to a single space-time point, i.e. constant map. To show that, we insert a product of $\delta$-functions $\prod_{\xi} \delta\left(X^{\mu}(\xi)-x^{\mu}\right)$ into the path integral, which singles out a constant map in the configuration space. The partition function reduces to that of two dimensional gravity with no matter, i.e. pure 2-d gravity theory

$$
Z_{x}^{\text {top }}=\int \mathcal{D} g \mathcal{D} X e^{-S[g, X]} \prod_{\xi} \delta\left(X^{\mu}(\xi)-x^{\mu}\right)=\int \mathcal{D} g e^{-S^{\prime}[g]}
$$

where the action $S^{\prime}$ is given by (2.2) without $X$ dependent terms.

In fact, the pure two dimensional gravity is described by a TG which can be thought of as $c=-2$ matter coupled with 2-d gravity [6] [8]. Therefore the configuration space of (2.1) contains configurations corresponding to one TG for every space-time point $x^{\mu}$.

More generally, the configuration space contains configurations which map regions, which will be denoted black regions, of world-sheet to single points in space-time and 
rest of world-sheet, denoted as white regions, to a surface in space-time. The region of the world-sheet mapped to single space-time point is described by TG with a suitable boundary condition. Therefore we divide the configuration space into three parts:

i). constant maps, topological sector.

ii). generic maps, string sector.

iii). mixed maps, interacting sector.

We will assume that all three sectors are important in our theory and their relative weights are governed by a yet undetermined coupling constant $\lambda$ which we will discuss in detail later.

String sector of the configuration space was considered solely in the usual formulation of string theory and one implicitly assumed the other two sectors had zero measure and therefore could be ignored. One of the reasons for such an assumption is that the string with single point as target space is trivial. Recent advances in $d \leq 1$ string theory show that this is not the case[3]. Therefore it is necessary to re-examine this question. Another possibility is that the non-perturbative effects in string theory amplify the contribution of these configurations and one can therefore put them in from beginning in perturbation theory. It is also conceivable that the theory is a total different string theory which is also certainly interesting.

The descriptions of i). and ii). are straightforward and therefore we will concentrate on iii). In string theory, the interesting observables are vertex operators integrated over the surface of the world-sheet. For these observables, the modifications in our theory come from contributions from the interacting sector. A general new contribution comes from a string diagram of colored surface Fig.1, and the path integral is performed in the the following way. For the white regions on the surface we integrate over $X$ with DBC and $g$ with proper gauge fixing. For the black regions we integrate only over $g$ with action $S^{\prime}$ in the proper gauge, and then integrate over the location $x$. The vertex operators will only be integrated over the white regions of the surface. The path integral can also be computed by inserting a product of $\delta$-functions for every point of the black region in (2.1), and then integrating over the different boundary values $x$ for each boundary as

$$
A\left(y_{1}, y_{2}, \cdots\right)=\int \mathcal{D} X \mathcal{D} g \prod_{i} d x e^{-S} \prod_{j} \delta\left(X\left(\xi_{j}\right)-y_{j}\right) \prod_{\xi \in \Sigma_{i}} \delta\left(X(\xi)-x_{i}\right)
$$

where $\Sigma_{i}$ ' are black regions of world-sheet. Because black regions are point-like in spacetime, the interactions are local in space-time. 
One can also view the black and white regions to represent two different types of strings, will be referred as string and topological string. In doing so, a colored surface represents interactions of strings and topological strings. This is analogy to the situation of interaction of different particles in first quantized theory. Therefore we associate a coupling constant $\lambda$ for each boundary between the two regions. This is the first example of a string theory with two types of strings interacting locally in space-time. It is also important to point out the interaction is not local on the world-sheet, which is certainly not a requirement in string theory.

Our main goal now is to develop a formalism to study the above theory in a systematic way. It is convenient to separate the problem into three parts. The white region(string part) is the well known object in string theory with suitable boundary condition. The black region(topological string part) is a TG with suitable boundary condition and the proper boundary conditions to glue those regions together.

What is the proper boundary condition? The answer actually depends on the particular gauge used. In conformal gauge, to maintain the BRST invariance one should require the matter stress-tensor to be continuous cross the boundary. On the string side, because the whole boundary is being mapped to a single point, the natural boundary condition is the DBC for $X^{\mu}$. The ghost fields are shared on both sides, and therefore they are continuous across the boundary. The correct procedure to implement these boundary conditions requires the introduction of auxiliary fields on the string part of the world sheet and will be addressed in a subsequent publication. We will see later that the conformal gauge is not the most efficient gauge to use in our theory for practical calculations. However, it is a good gauge to use to study the formal properties of the theory.

The main results of TG relevant for our purposes can be summarized as following[6]. In the conformal field theory description of TG, one starts with spin-2 bosonic ghost system together with the fermionic ghost with action

$$
S=S_{b c}+S_{\beta \gamma}=\frac{1}{\pi} \int b \bar{\partial} c+\bar{b} \partial \bar{c}+\beta \bar{\partial} \gamma+\bar{\beta} \partial \bar{\gamma}
$$

It is more convenient to bosonize the $\beta-\gamma$ ghost by

$$
\beta=e^{-\phi(z)} \partial \xi, \quad \gamma=e^{\phi(z)} \eta
$$

following Friedan, Martinec and Shenker [9]. The cosmological constant term is expressed in term of "Liouville" field $\phi$ as

$$
S_{\text {cosmo }}=-\frac{1}{\pi} \int \sqrt{\hat{g}} e^{-\phi(z, \bar{z})}
$$


One can further bosonize $\eta-\xi$ system, $\eta=e^{i \psi}, \xi=e^{-i \psi}$ and the dressed primary fields which will be our observables are

$$
O_{n}=e^{i n \psi(z, \bar{z})} e^{(n-1) \phi(z, \bar{z})}
$$

The correlation functions of these operators are not the main focal point here. The corrections to the amplitude of these operators due to the interacting sector can also be addressed by the formalism discussed below. Of course, in that case, we should sum over the contributions of the white regions instead.

\section{Modification of String Theory}

It turns out the simplest gauge to work is the GGRT [10] light-cone gauge. The first quantized string is formulated in terms of coordinates $X^{\mu}(\sigma, t)$ satisfying two dimensional wave equation

$$
\left(\frac{\partial^{2}}{\partial t^{2}}-\frac{\partial^{2}}{\partial \sigma^{2}}\right) X^{\mu}(\sigma, t)=0
$$

which sweep out a two dimensional surface. The reparametrization invariance of the world sheet implies the phase space of the string is subject to the first class constraints

$$
\begin{aligned}
\dot{X}^{2}+X^{\prime 2} & =0, \\
\dot{X} \cdot X^{\prime} & =0 .
\end{aligned}
$$

In the light-cone gauge a relativistic string $X^{\mu}(\sigma)$ can be represented in terms of only its transverse components, and hence the question of ghost never appears. Their constraints imply $X^{+}=\frac{1}{\sqrt{2}}\left(X^{0}+X^{D-1}\right)=\tau$ independent of $\sigma$. All coordinates are determined in terms of physical operators $\mathbf{X}(\sigma)$, "transverse" components of $X^{\mu}$, i.e. $\mu=1,2, \cdots, D-2$ and thereby eliminating the redundant longitudinal modes. In the light-cone gauge, the matter stress-energy tensors are set to zero by the constraint condition (3.2). Another advantage of the light-cone gauge is that the only consistent closed string field theory is formulated in this gauge.

On the normal string part, the boundary condition is given by $\prod_{s} \delta(\mathbf{X}(s)-\mathbf{x})$, and the value of $X^{+}$along the boundary. On the topological string part, we will work in the conformal gauge. The fields $\beta-\gamma$ and $b-c$ of the TG are restricted to the black region of the surface and therefore the correct boundary condition is the Neumann boundary 
condition for both $\beta-\gamma$ and $b-c$. That is the boundary condition we are going to use for the black region. We will refer this gauge choice as "mixed gauge".

It is assumed in critical bosonic string theory that the Liouville field decouples from the theory due to Weyl invariance. The Liouville action is multiplied by $D-26$, which vanishes in the critical dimension. Therefore it is clear in our case that the boundary condition for the Liouville field of the TG is the Neumann boundary condition. The boundary condition of the Liouville field will be very different if the white region of the theory is not in the critical dimension.

In the mixed gauge, the contribution of many string diagrams with the black region that have the same number of boundaries but different genus, moduli, etc. can be computed together by using the matrix model method 11]. The main advantage of the matrix model method is that one can evaluation the integral

$$
W^{(n)} \sim \sum_{\Sigma_{g}^{n}} \int \mathcal{D} g e^{-S^{\prime}}
$$

directly, where the sum is over all possible surfaces with $n$ boundaries. First we compute the sum over all surfaces with $n$ macroscopic boundaries of fixed lengths in TG. It is given by 11$]$

$$
\begin{aligned}
W_{l}^{(n)}|\ln \Delta| & =(-1)^{n+1}|\ln \Delta| 2^{3-n} \mu^{n / 2}\left(-96 \bar{\kappa}^{2}\right)^{-1+\frac{n}{2}} \\
& \times \sum_{g=0}^{\infty} \frac{\bar{\kappa}^{2 g}}{g !}(l \sqrt{\mu})^{3 g-3+n} I_{3 g-3+n}(l \sqrt{\mu})
\end{aligned}
$$

where $l=\sum l_{i}$ is the total length of the boundaries. $\mu$ the renormalized cosmological constant and $\bar{\kappa}^{2}=\kappa^{2} / \mu^{3}$ is the dimensionless loop counting parameter. $\kappa$ is the the renormalized string coupling constant of TG and $I$ are the modified Bessel functions.

Notice that $W_{l}^{(n)}$ depends only on the total length of the boundaries. Summation over all possible boundary lengths can be easily done by introducing a boundary cosmological constant $\xi$

$$
W^{(n)}=\int d l_{1} \cdots d l_{n} e^{-\xi l} W_{l}^{(n)}
$$

$W^{(n)}$ is a function of $\mu, \xi, \bar{\kappa}^{2}$ and contains contribution of all possible surface with $n$ boundaries.

For each boundary of black surface we glue to it a boundary of white surface. The incredibly huge amount of string diagrams are greatly simplified. In space-time the contributions of topological string correspond to introducing new point-like interactions in 
string theory. Unfortunately there are still a very large number of string diagrams to be considered. One also expects that the interesting phenomena come from a partial (infinite) summation over contributions from these diagrams. The first quantized formalism is not very good at handling this question efficiently. In the next section we will discussion how to incorporate these diagrams in the second quantized string field theory via new string interactions.

\section{Light-Cone String Field Theory}

We start by summarizing the main results of the light-cone bosonic string field theory. In this gauge, the string field functional $\Psi[X(\sigma)]$ is independent of the longitudinal components except the zero modes of $X^{+}, X^{-}$. One then takes the Fourier transform with respect to the zero mode of $X^{-}$, so the field depends on $P^{+}$, the width of the string. The string field functional becomes $\Psi_{P^{+}}[\mathbf{X}, \tau]$. The region of the parameterization of the string is $\left[0, \pi P^{+}\right]$. The Lagrangian for the closed bosonic string field theory [12 follows simply from that of light-cone open string field theory [1] 13 to reproduce the results of the Mandelstam's interacting string formalism[14]. The Lagrangian is given by

$$
\mathcal{L}=\mathcal{L}_{0}+\mathcal{L}_{1}=\Psi^{\dagger} K \Psi+\frac{1}{2} g\left(\Psi^{\dagger} \circ \Psi \circ \Psi+\text { h.c. }\right)
$$

in the operator formalism. The quadratic term will reproduce the first-quantized Schrödinger equation

$$
i \frac{\partial \Psi_{P^{+}}}{\partial \tau}[\mathbf{X}, \tau]=\frac{\pi}{2} \int_{0}^{\pi P^{+}}\left[-\frac{\delta^{2}}{\delta \mathbf{X}(\sigma)^{2}}+\left(\frac{\mathbf{X}^{\prime}(\sigma)}{\pi}\right)^{2}\right] \Psi_{P^{+}}[\mathbf{X}, \tau] \equiv h \Psi_{P^{+}}[\mathbf{X}, \tau]
$$

The o operation in three string interaction Lagrangian $\mathcal{L}_{1}$ is defined by the geometrical three string interaction vertex.

The modification of the string field theory from the point-like interactions, which describe the contributions of the topological strings, can be accomplished by adding new interaction terms, in fact infinitely many of them, to the bosonic string Lagrangian. On the quadratic level, there are two new interacting terms that are obtained by looking at the first quantized amplitude with two vertex operators inserted. They are

$$
\mathcal{L}_{2}^{(1)}+\mathcal{L}_{2}^{(2)}=\Psi^{\dagger} \mathcal{O}_{1} \Psi+\Psi^{\dagger} \mathcal{O}_{2} \Psi
$$


as illustrated in Fig.2. $\mathcal{L}_{2}^{(1)}$ represents an interaction where at given $X^{+}$a segment of string carrying finite $P^{+}$becomes transversely localized, i.e. $\mathbf{X}(\sigma)=\mathbf{x}$ along the segment. It has been shown by Green and Shapiro[7] that such an interaction is point-like in space-time and therefore Lorentz invariant. $\mathcal{L}_{2}^{(1)}$ is also proportional to $W^{(1)}$ to take into account the contributions of all black regions with one boundary. We also introduce a new coupling constant $\lambda$, and its power counts the number of boundaries for the interaction. Of course, we have to sum over all possible such interactions by integrating over the length and the locations of the segment on the string.

It is important to point out that we also have to integrate over the locations of the interaction over space-time. In doing so, we can ignore the $\ln |\Delta|$ by interpreting it as the density of topological strings in space-time. In the matrix model, $\Delta$ is introduced as energy gap which is taken to zero in the double scaling limit. Similar treatment will be done for other interactions.

$\mathcal{L}_{2}^{(2)}$ is the interaction where the whole string becomes transversely localized at some instant $X^{+}$. Summing over the contributions from all black surfaces with two boundaries and putting in the proper power of coupling constant $\lambda$, it is given explicitly by

$$
\begin{aligned}
\mathcal{L}_{2}^{(2)} & =\frac{1}{2} \lambda^{2} W^{(2)} \int \prod_{i=1}^{2} d P^{+i} \mathcal{D} \mathbf{X}_{i}(\sigma) d \mathbf{x} \delta\left(P_{2}^{+}-P_{1}^{+}\right) \Psi_{P_{2}^{+}}^{\dagger}\left[\mathbf{X}_{2}(\sigma), \tau\right] \Psi_{P_{1}^{+}}\left[\mathbf{X}_{1}(\sigma), \tau\right] \\
& \times \prod_{\sigma} \delta\left(\mathbf{X}_{2}(\sigma)-\mathbf{x}\right) \delta\left(\mathbf{X}_{1}(\sigma)-\mathbf{x}\right)+h . c
\end{aligned}
$$

Few remarks are in order before we go on to describe higher order interactions. The interaction $\mathcal{L}_{2}^{(1)}$ was considered by Green and Shapiro [7], and later Green [15] as the emission of zero momentum scalar particles into vacuum. They showed that the interaction is truly point-like in space-time and that it can modify the large-angle elastic scattering in the open string when considering the leading contribution to the scattering amplitude. In their original formalism, $\mathcal{L}_{2}^{(2)}$ is negligible because it is the limiting case of $\mathcal{L}_{2}^{(1)}$ when the whole string becomes transversely localized.

In our formulation, the interactions are consequences of normal strings interacting with topological strings. Because the interactions are geometric, the consistent condition should be much easier to check. In particular $\mathcal{L}_{2}^{(2)}$ takes into account infinitely many surfaces with two boundaries which are different surfaces from those of $\mathcal{L}_{2}^{(1)}$, and therefore must be included. In fact, the first quantized picture tells us exactly what the new interaction terms are in a given order of string field theory. 
There are new cubic interaction terms in our theory, $\mathcal{L}_{3}^{(2)}+\mathcal{L}_{3}^{(3)}$, which are represented in Fig.3. The four-string interacting terms are $\mathcal{L}_{4}^{(2)}+\mathcal{L}_{4}^{(3)}+\mathcal{L}_{4}^{(4)}$, as shown in Fig. 4 .

It is very important to note that for every boundary there are at most two light-cone strings simultaneous touching it. This limits the number of interactions a black region with $n$ boundaries can generate, e.g. there are new interaction terms proportional to $W^{(n)}$ which couple from $n$ to $2 n$ strings. In particular there are only a finite number of new diagrams for $N$ string tree amplitude when we use the correct propagator $\left(K+\mathcal{O}_{1}+\mathcal{O}_{2}\right)^{-1}$ from the quadratic part of the theory.

The propagator $\left(K+\mathcal{O}_{1}+\mathcal{O}_{2}\right)^{-1}$ summarizes the contributions from infinitely many string diagrams. Because $W^{(n)} \propto(-1)^{n+1}$, the effect of $\mathcal{O}_{1}$ and $\mathcal{O}_{2}$ could shift the masses square upward for real coupling constant $\lambda$. The determination of the mass spectrum of our theory will be addressed in a future publication.

\section{Conclusions and Remarks}

One of the obvious questions is whether the new terms included in string field theory preserve the unitarity of the string amplitudes. The interaction of strings with pointlike structure has been studied [7] [15]. The major difference is that we give an explicit realization of the point-like structure, i.e. induced by TG. Therefore the properties of the point-like structure are determined. The point-like structure couples with a segment of string and the coupling is geometric in the two dimensional world-sheet. Nevertheless Green's[15] discussion about the unitarity of scattering amplitude still applied. Our theory is at least perturbative unitarity.

What is the fundamental reason for the existence of the mixed sector of the string theory? The following scheme is one possible answer to this question. As we mentioned early, the configuration space divided into three sectors. At very high energy, or high temperature, the whole theory is given by the topological sector. The theory consists of product of TG for each space-time point. For each TG, there is a BRST symmetry(string symmetry) and the symmetry of the theory is the product of these BRST symmetries. Moreover, the theory is topological in space-time and has no continuous degree of freedom in the normal sense. At lower energy the physics is given by the theory we described in the main text and the symmetry, when we work in conformal gauge, is one BRST symmetry. The natural question to ask is where are all the continuous degrees of freedom come from? We expect the partial answer is that they coming from symmetry breaking. The exact 
dynamical question of how this process occurs is not clear. The theory we describe is only an effective theory after symmetry breaking.

The extension of our results to superstring is of considerable interest. Instead of topological gravity, we should consider topological supergravity as the topological sector of superstring. By including the topological sector and interacting sector to superstring, it is conceivable that the theory automatically gives an expectation value to dilaton and breaks supersymmetry. The reason is that the point-like structure from the topological string couples only with scalar fields of superstring. Clearly, more work in this direction is needed to clarify this issue.

The mixed sector is also playing a crucial role when considering the non-critical string theory of $d>1$. In this case the light-cone gauge for the string sector is not well understood and the gauge to work in is the conformal gauge. The boundary condition of the Liouville field is the central issue. When we use the prescription of the Liouville field in terms of a free field, with suitable coupling to the curvature 16, the boundary condition is simply the continuation condition of the Liouville field. The scaling behavior of the partition function, $Z(\mu) \sim \mu^{2-\gamma}$, can be easily calculated [17] as in [18] with

$$
\gamma=2-\sqrt{\frac{25-d}{3}}
$$

for the spherical topology.

It is reasonable to expect that the topological sector of the theory describes the theory at higher temperature. If we take the point of view that the the statistical mechanics of string theory at finite temperature is described also by Euclidean string theory with the time direction compactified on a circle[2], then at very high temperatures, above KosterlitzThouless (KT) transition temperature, the theory is a collection of TG theories. When we go below the KT transition temperature, the high temperature string theory will be described by collection of one dimensional (the "time" direction) strings for every space point. In this case the free energy per unit volume can be computed by using the matrix model technique. It is given[19], at least when the the temperature is low enough, by

$$
\frac{f}{T} \sim \frac{1}{4 \pi} \frac{1}{T g_{s t}^{2}}-\frac{1}{36 \pi}\left(\pi^{2} T+\frac{1}{T}\right)+\cdots
$$

which can be written in a more suggestive form when we use the effective string coupling constant $g_{\text {eff }}=T g_{s t}$ as

$$
\frac{f}{T} \sim \frac{1}{4 \pi} \frac{T}{g_{\text {eff }}^{2}}-\frac{1}{36 \pi}\left(\pi^{2} T+\frac{1}{T}\right)+\cdots,
$$


where we absorb the $\ln \mu$ into the definition of volume as before. It has precisely the same form as suggested by Atick and Witten[2], from a string calculation. The dominant contribution is from genus zero. It therefore offers another piece of evidence that the high energy phase of strings is described by topological string theory.

\section{Acknowledgments}

I would like to thank P. Griffin, M. McGuigan S. Sin, and especially C. Thorn for valuable discussions. This work was supported in part by the United States Department of Energy under contract No. FG05-86-ER40272. 


\section{References}

[1] e.g. M. B. Green, J. H. Schwarz and E. Witten, "Superstring Theory", Cambridge University Press 1987.

[2] J. Atick and E. Witten, Nucl. Phys. B310 (1989)291.

[3] V. Knizhnik, A. A. Polyakov and A. Zamolodchikov, Mod. Phys. Lett. A3 (1988) 819.

[4] E. Brézin and V. A. Kazakov, Phys. Lett. 236B (1990) 14; M. R. Douglas and S. H. Shenker, Nucl. Phys. B335 91990) 635; D. J. Gross and A. A. Migdal, Phys. Rev. Lett. 64 (1990) 127.

[5] E. Witten, Nucl Phys. B340 (1990) 281.

[6] J. Distler, Nucl Phys. B342 (1990) 523.

[7] M.B. Green and J.A. Shapiro, Phys. Lett. 64B (1976) 454; M.B. Green, Phys. Lett. 69B (1977) 89.

[8] E. Verlinde and H. Verlinde, Nucl. Phys. B348 (1991) 457.

[9] D. Friedan, E. Martinec and S. Shenker, Nucl.Phys. B271 (1986) 93.

[10] J. Goldstone, P. Goddard, C. Rebbi and C. Thorn, Nucl Phys. B56 (1973) 205.

[11] J. D. Edwards and I. R. Klebanov, Mod. Phys. Lett. A6 (1991) 2901; G. Moore Nucl. Phys. B377 (1992) 143.

[12] C. B. Thorn, Nucl. Phys. B263 (1986) 493.

[13] M. Kaku and K. Kikkawa, Phys. Rev. D10 (1974) 1110; M. Kaku and K. Kikkawa, Phys. Rev. D10 (1974) 1823. E. Cremmer and J. L. Gervais, Nucl. Phys. B90 (1975) 410.

[14] S. Mandelstam, Nucl. Phys. B64 (1973) 205; B69 (1974) 77; B69 (1974) 109.

[15] M. B. Green, Nucl. Phys. B116 (1976) 449; B124 (1977) 461.

[16] T.L. Curtright and C.B. Thorn, Phys. Rev. Lett. 48 (1982) 1309.

[17] Z. Qiu, to appear.

[18] F. David Mod. Phys. Lett. A3 (1988) 1651; J. Distler and H. Kawai, Nucl. Phys. B321 (1988) 509.

[19] D. J. Gross and I. R. Klebanov, Nucl. Phys. B344 (1990) 475. 